\title{
Predictors of Discontinuation of Antipsychotic Therapy in Patients with Acute Schizophrenia: A 1-Year Observational Study with More Than 1000 Patients
}

\author{
Michihiro Takahashi', Shinji Fujikoshi², Jumpei Funai', Levent Alev ${ }^{4}$, Masaomi Iyo ${ }^{5}$ \\ ${ }^{1}$ Takahashi Psychiatric Clinic, Ashiya, Japan \\ ${ }^{2}$ Statistical Science, Lilly Research Laboratories Japan, Eli Lilly Japan K.K., Kobe, Japan \\ ${ }^{3}$ Science Communications, Lilly Research Laboratories Japan, Eli Lilly Japan K.K., Kobe, Japan \\ ${ }^{4}$ Medical Science, Lilly Research Laboratories Japan, Eli Lilly Japan K.K., Kobe, Japan \\ ${ }^{5}$ Department of Psychiatry, Graduate School of Medicine, Chiba University, Chiba, Japan \\ Email: alev levent@lilly.com
}

Received 15 April 2014; revised 18 May 2014; accepted 12 June 2014

Copyright (C) 2014 by authors and Scientific Research Publishing Inc.

This work is licensed under the Creative Commons Attribution International License (CC BY). http://creativecommons.org/licenses/by/4.0/

(c) (i) Open Access

\begin{abstract}
Discontinuation of antipsychotic therapy has been a significant clinical issue among patients with schizophrenia, since the patients who discontinued antipsychotic treatment showed worse clinical and functional outcomes, and higher risks of relapse of schizophrenia symptoms and hospitalization. We conducted a post-hoc analysis of a post-marketing research with a 12-month follow-up period to identify the predictors for discontinuation of antipsychotic monotherapy in Japan. This is a prospective, naturalistic multicenter observational study, designed to evaluate the discontinuation rates of olanzapine monotherapy and non-olanzapine antipsychotic monotherapy in Japanese adult patients with acute schizophrenia. Patients were treatment-naïve, or had switched from other antipsychotics or from poly-pharmacotherapy to oral antipsychotic monotherapy. We analyzed the correlation of discontinuation of antipsychotic monotherapy with baseline characteristics of patients. A total of 1089 patients (578 patients treated with olanzapine and 511 with non-olanzapine antipsychotics) were eligible for analysis. By the end of the 12-month study period, 614 patients $(56.4 \%)$ discontinued antipsychotic therapy. Multivariate logistic regression analyses indicated significantly lower discontinuation rates in all patients treated with antipsychotics: older age (Odds ratio [OR], $0.871 ; 95 \%$ confidence interval $[\mathrm{CI}], 0.797$ to $0.953 ; p=0.003$ ), outpa-
\end{abstract}

*Corresponding author.

How to cite this paper: Takahashi, M., Fujikoshi, S., Funai, J., Alev, L. and lyo, M. (2014) Predictors of Discontinuation of Antipsychotic Therapy in Patients with Acute Schizophrenia: A 1-Year Observational Study with More Than 1000 Patients. Open Journal of Psychiatry, 4, 364-371. http://dx.doi.org/10.4236/ojpsych.2014.44042 
tient status (OR, $0.508 ; 95 \% \mathrm{CI}, 0.383$ to 0.675 ; $\mathrm{p}<0.001)$, prior use of antipsychotics $(0 \mathrm{R}, 0.693$; $95 \% \mathrm{CI}, 0.516$ to $0.930 ; \mathrm{p}=0.015)$, and olanzapine group showed lower discontinuation rate than that of non-olanzapine group (OR, 1.416; 95\% CI, 1.086 to $1.846 ; p=0.010$ ). The present study indicated that the outpatient status, older age, and prior use of antipsychotics have better adherence to antipsychotic treatment. In addition to these factors, use of anti-parkinson agents showed lower discontinuation rates in the olanzapine monotherapy group.

\section{Keywords}

Predictor of Discontinuation, Antipsychotic Monotherapy, Long-Term Observational Study, Schizophrenia

\section{Introduction}

Schizophrenia is a mental illness that involves a range of cognitive, behavioral, and emotional dysfunctions, as well as social and occupational dysfunctions [1]. Atypical antipsychotics have been widely used as a first-line therapy for the acute and maintenance phases of schizophrenia. Although a number of antipsychotics are available, monotherapy remains an important option due to lack of sufficient evidence for the efficacy and safety of poly-pharmacotherapy.

Discontinuation of antipsychotic therapy has been a significant clinical issue among patients with schizophrenia. Compared with patients who continued treatment with antipsychotics, patients who discontinued antipsychotic treatment showed worse clinical and functional outcomes and higher risks of relapse of schizophrenia symptoms and hospitalization [2]-[5]. In recent years, a number of studies conducted outside of Japan have investigated the predictors of antipsychotic adherence [3] [6]-[11].

We conducted this post-hoc analysis of a prospective, multicenter observational study to investigate the relationship between baseline characteristics of patients and discontinuation of antipsychotic monotherapies to identify the predictors for discontinuation in Japanese patients with acute schizophrenia.

\section{Methods}

\subsection{Study Design and Patients Selection}

This is a prospective, naturalistic multicenter observational study with 12-month follow-up, designed to evaluate the discontinuation rates of olanzapine monotherapy and non-olanzapine antipsychotic monotherapy in Japanese adult patients with acute schizophrenia in a routine clinical practice setting. The key eligibility criteria for this study were patients at least 20 years of age who were diagnosed with schizophrenia according to the Diagnostic and Statistical Manual of Mental Disorders fourth Edition (DSM-IV) Text Revision with a Clinical Global Impression-Severity of Illness (CGI-S) Schizophrenia score of $\geq 4$ at the start of monotherapy, and had acute symptoms developed within one month prior to the start of oral antipsychotic monotherapy. Patients were treatment-naïve, or had switched from other antipsychotics or from poly-pharmacotherapy to oral antipsychotic monotherapy. All patients provided informed consent before starting any study procedures, and this study was conducted in compliance with Helsinki Declaration and the guideline of Good Post-marketing Study Practice (GPSP).

\subsection{Measurements}

Patients were monitored until discontinuation of the study treatment. In this study, reasons for discontinuation included discontinuation of treatment with antipsychotic monotherapy, additional use of any other antipsychotics, lost to follow-up, discontinuation of treatment by request of the patient or decision of the investigator. We analyzed the correlation of discontinuation of antipsychotic monotherapy with baseline characteristics of patients (gender, age, body mass index [BMI], onset of illness, outpatient or inpatient status at the start of study treatment, history of schizophrenia, living status, prior use of antipsychotics, CGI-S Schizophrenia score, Brief Psychiatric Rating Scale [BPRS] score, use of concomitant medications [i.e., anti-Parkinson agents, antidepres- 
sants, anti-anxiety/sleep agents, antiepileptic agents, mood-stabilizers] at the start of monotherapy).

\subsection{Statistical Analysis}

The data were analyzed in all patients treated with antipsychotics, and patients with olanzapine monotherapy and with non-olanzapine antipsychotic monotherapy. Differences in baseline patient characteristics between patients who discontinued antipsychotic monotherapy before completion of the study period and those who continued antipsychotic monotherapy for 12 months were compared using Student's t-test, Fisher's exact test, or the Monte Carlo method. Multivariate logistic regression analyses were used to calculate odds ratios (OR) and 95\% confidence intervals (CI) to determine baseline variables that predicted the likelihood of discontinuation of antipsychotic monotherapy. Univariate analyses were conducted for all collected baseline items. However, multivariate analyses were conducted only for those baseline items with no or few missing data. All statistical tests were conducted based on a 2-sided significance level of 0.05 .

\section{Results}

This study enrolled 1124 patients from 72 centers in Japan and was conducted from January 2010 to August 2012. Of the enrolled patients, 35 patients were excluded: 9 due to lack of case report, 14 who were under 20 years of age, and 12 for concomitant use of multiple antipsychotics. A total of 1089 patients (578 patients treated with olanzapine and 511 with non-olanzapine antipsychotics) were eligible for analysis. In the non-olanzapine antipsychotic monotherapy group, atypical antipsychotics were administered to 487 patients (160 risperidone, 154 aripiprazole, 44 quetiapine, 67 blonanserin, 40 paliperidone, 16 perospirone, and 6 zotepine) and typical antipsychotics were administered to 24 patients (13 haloperidol, 4 bromperidol, 3 sulpiride, 2 chlorpromazine, 1 levomepromazine, and 1 fluphenazine). By the end of the 12-month study period, 614 patients (56.4\%) discontinued antipsychotic monotherapy; 310 patients (53.6\%) in the olanzapine monotherapy group and 304 patients (59.5\%) in the non-olanzapine antipsychotic monotherapy group. The baseline characteristics of patients including those patients who discontinued the antipsychotic monotherapy and those who continued throughout the study period in both groups are shown in Table 1.

In all 1089 patients, discontinued patients were significantly more likely to be younger $(p<0.001)$, inpatient status $(\mathrm{p}<0.001)$, with a higher CGI-S Schizophrenia score $(\mathrm{p}=0.022)$, a lower BPRS Negative score $(\mathrm{p}<$ 0.001), a lower BPRS Anxiety/Depression score ( $<0.001)$, and a shorter history of schizophrenia $(p=0.039)$. In the 578 olanzapine monotherapy patients, discontinued patients were significantly more likely to be younger ( $p<0.001)$, be an inpatient ( $p=0.005)$, have a shorter history of schizophrenia $(p=0.002)$, not to have previously used antipsychotics $(\mathrm{p}<0.001)$, have higher CGI-S Schizophrenia score $(\mathrm{p}=0.022)$, have lower BPRS Negative score $(p<0.001)$, and not to have used anti-Parkinson agents $(p=0.005)$. In the 511 non-olanzapine antipsychotic monotherapy patients, discontinued patients were significantly more likely to be an inpatient ( $<$ 0.001), have lower BPRS Negative score $(p=0.039)$, have lower BPRS Anxiety/Depression score $(p<0.001)$, not to have used antidepressants $(p=0.022)$, and not to have used anti-anxiety/sleep agents $(p=0.048)$. For other baseline characteristics variables, there was no significant difference between discontinued and continued patients.

The results of multivariate logistic analyses are summarized in Table 2. In all patients treated with antipsychotics, an older age (OR, 0.871; 95\% CI, 0.797 to 0.953; $\mathrm{p}=0.003$ ), outpatient status (OR, 0.508; 95\% CI, 0.383 to $0.675 ; \mathrm{p}<0.001$ ), and prior use of antipsychotics (OR, 0.693; 95\% CI, 0.516 to $0.930 ; \mathrm{p}=0.015$ ) were significantly less likely to discontinue antipsychotic monotherapy. In the olanzapine monotherapy group, patients with older age $(p=0.008)$, outpatient status $(p=0.020)$, prior use of antipsychotics $(p=0.004)$, and use of anti-Parkinson agents $(\mathrm{p}=0.050)$ were significantly less likely to discontinue olanzapine monotherapy. In the non-olanzapine antipsychotic monotherapy group, only outpatient status $(\mathrm{p}<0.001)$ showed a significantly lower risk of discontinuation of antipsychotic monotherapy. Figure 1 indicates the discontinuation odds ratios for all antipsychotics and each treatment group.

\section{Discussion}

The present study is a large prospective, naturalistic multicenter observational study that included Japanese patients with acute schizophrenia and provided important information on the baseline characteristics of patients which could predict continuation of the antipsychotic monotherapy. 
Table 1. Baseline characteristics of patients.

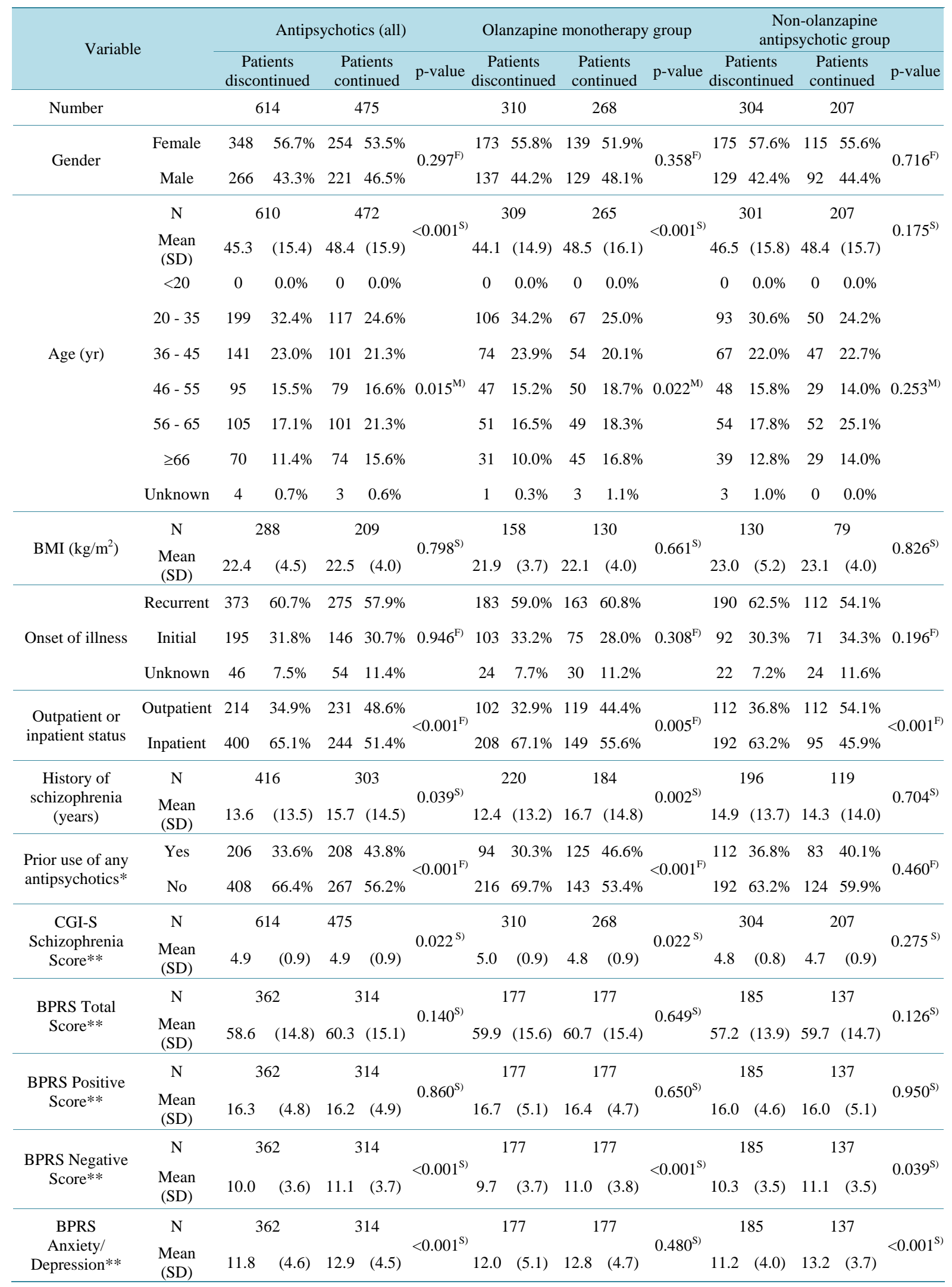




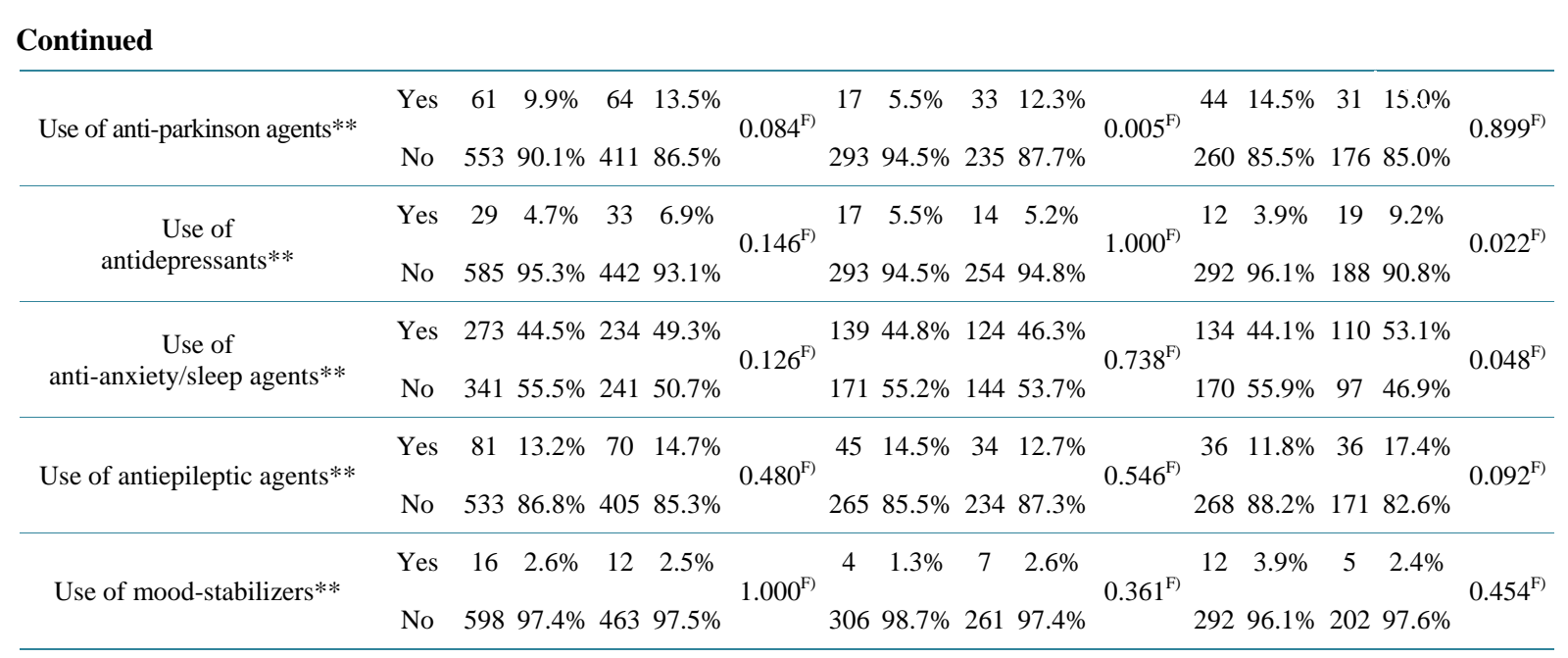

*Within 1 month before initiation of monotherapy; **At initiation of monotherapy. CGI-S: Clinical Global Impression-Severity of Illness; BPRS:

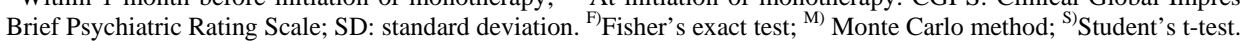

Table 2. Discontinuation odds ratios by baseline characteristics (multivariate logistic regression analyses).

\begin{tabular}{|c|c|c|c|c|c|c|c|c|c|}
\hline \multirow[t]{2}{*}{ Factor (test/reference) } & \multicolumn{3}{|c|}{ All Antipsychotics } & \multicolumn{3}{|c|}{ Olanzapine group } & \multicolumn{3}{|c|}{ Non-olanzapine group } \\
\hline & OR & $95 \%$ CI & p-value & OR & $95 \%$ CI & p-value & OR & $95 \%$ CI & p-value \\
\hline Gender (female/male) & 1.204 & $0.920-1.576$ & 0.175 & 1.163 & $0.803-1.686$ & 0.424 & 1.370 & $0.913-2.056$ & 0.129 \\
\hline Age (units = 10 years old) & 0.871 & $0.797-0.953$ & 0.003 & 0.843 & $0.743-0.957$ & 0.008 & 0.916 & $0.804-1.044$ & 0.187 \\
\hline Onset of illness (recurrent/initial) & 1.145 & $0.854-1.535$ & 0.366 & 0.994 & $0.664-1.488$ & 0.976 & 1.294 & $0.836-2.004$ & 0.247 \\
\hline $\begin{array}{l}\text { Outpatient or inpatient status } \\
\text { (outpatients/inpatients) }\end{array}$ & 0.508 & $0.383-0.675$ & $<0.001$ & 0.622 & $0.416-0.929$ & 0.020 & 0.398 & $0.264-0.601$ & $<0.001$ \\
\hline $\begin{array}{l}\text { Prior use of any antipsychotics* } \\
\text { (yes/no) }\end{array}$ & 0.693 & $0.516-0.930$ & 0.015 & 0.549 & $0.366-0.824$ & 0.004 & 0.886 & $0.568-1.383$ & 0.595 \\
\hline $\begin{array}{l}\text { CGI-S Schizophrenia Score** } \\
\text { (units }=1 \text { ) }\end{array}$ & 1.049 & $0.900-1.223$ & 0.540 & 1.078 & $0.879-1.322$ & 0.468 & 1.058 & $0.830-1.348$ & 0.650 \\
\hline $\begin{array}{l}\text { Use of anti-parkinson agents** } \\
\text { (yes/no) }\end{array}$ & 0.844 & $0.553-1.288$ & 0.431 & 0.500 & $0.250-0.999$ & 0.050 & 1.155 & $0.644-2.071$ & 0.629 \\
\hline Use of antidepressants** (yes/no) & 0.827 & $0.472-1.448$ & 0.506 & 1.243 & $0.553-2.792$ & 0.599 & 0.576 & $0.252-1.315$ & 0.190 \\
\hline $\begin{array}{c}\text { Use of anti-anxiety/sleep agents** } \\
\text { (yes/no) }\end{array}$ & 0.860 & $0.653-1.133$ & 0.285 & 0.988 & $0.679-1.437$ & 0.950 & 0.707 & $0.463-1.079$ & 0.108 \\
\hline $\begin{array}{l}\text { Use of antiepileptic agents** } \\
\text { (yes/no) }\end{array}$ & 1.088 & $0.733-1.614$ & 0.677 & 1.719 & $0.971-3.044$ & 0.063 & 0.821 & $0.457-1.475$ & 0.509 \\
\hline $\begin{array}{c}\begin{array}{l}\text { Use of mood-stabilizing drugs** } \\
\text { (yes/no) }\end{array}\end{array}$ & 0.987 & $0.420-2.322$ & 0.977 & 0.335 & $0.076-1.481$ & 0.149 & 1.837 & $0.551-6.128$ & 0.323 \\
\hline $\begin{array}{c}\text { Treatment } \\
\text { (Non-olanzapine/Olanzapine) }\end{array}$ & 1.416 & $1.086-1.846$ & 0.010 & & & & & & \\
\hline
\end{tabular}

*Within 1 month before initiation of monotherapy; **At initiation of monotherapy. CGI-S: Clinical Global Impression-Severity of Illness; OR: Odds ratio; $\mathrm{CI}$, confidence interval. $\mathrm{OR}>1$ indicates that test item has higher risk of discontinuation of antipsychotic monotherapy than reference item.

Previous studies conducted outside of Japan have also identified some general predictors for continuation of antipsychotic therapy. Older age [3] [6] [8]-[11], male gender [6]-[8], and longer history of schizophrenia [9] were similarly identified to be associated with continuation of antipsychotic therapy. On the other hand, our results were that in the univariate analysis, older age, lower CGI-S Schizophrenia score, higher BPRS Negative score, higher BPRS Anxiety/Depression score, outpatient status, and longer history of schizophrenia were identified as a predictor of continuation. In multivariate analysis, an older age, outpatient status, and prior use of antipsychotics were significantly likely to continue antipsychotic monotherapy. 

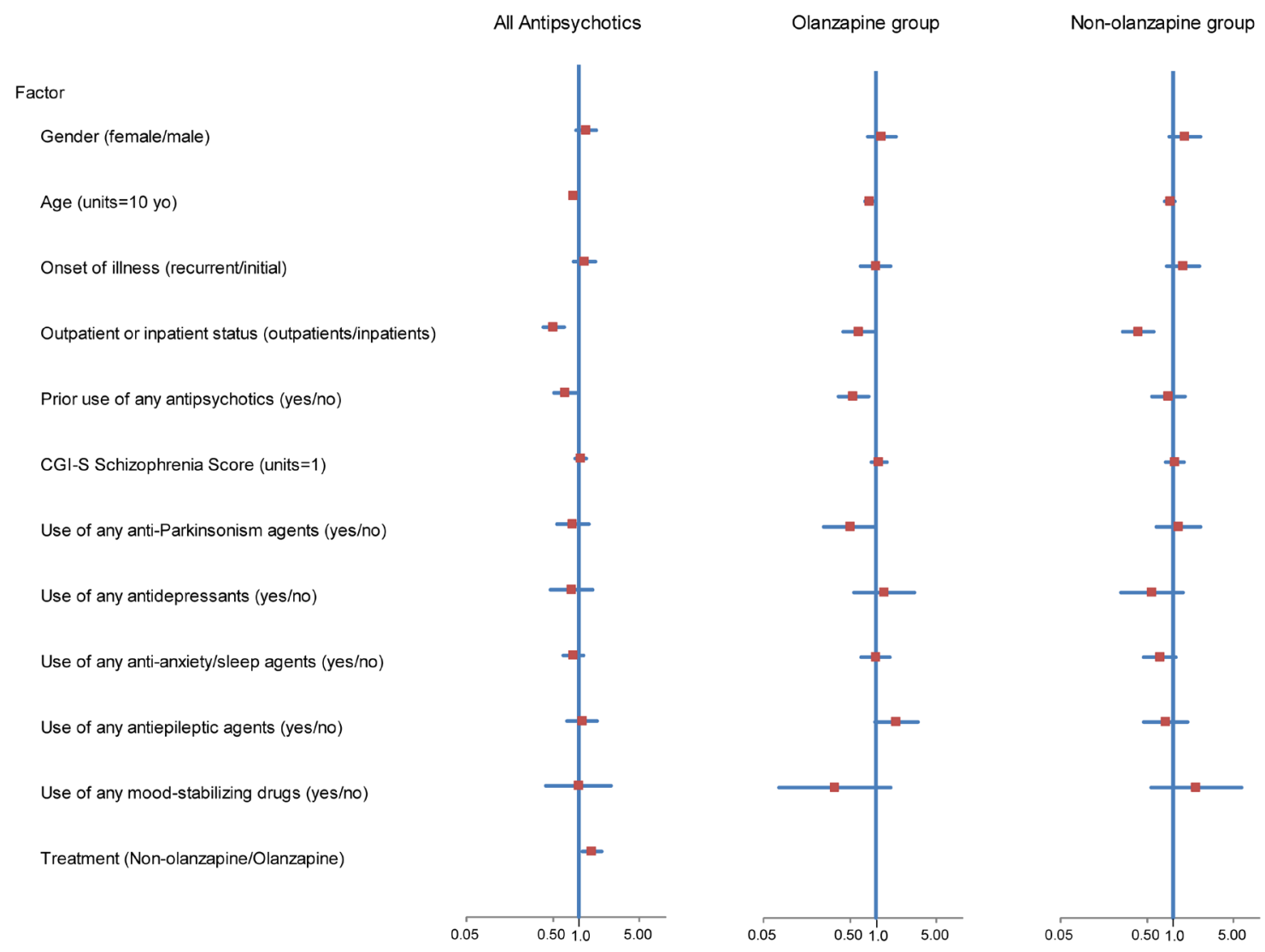

Figure 1. Discontinuation odds ratios by baseline characteristics.

Olanzapine showed higher continuation rates in several reports [12]-[15] and predictors of continuation of olanzapine treatment would be beneficial in a clinical practice. Older age, prior use of antipsychotics, and use of anti-Parkinson agents were identified as predictors of continuing with only olanzapine monotherapy. First of all, patients with $\geq 66$ years of age administered olanzapine monotherapy were more likely to continue treatment. Although antipsychotics should be administered to older patients with caution, olanzapine could be a better therapeutic option for older patients with acute schizophrenia due to better treatment adherence. Secondly, patients administered olanzapine monotherapy who previously used antipsychotics or were using anti-Parkinson agents at the start of monotherapy were more likely to continue antipsychotic monotherapy. For those patients having insufficient efficacy, low treatment adherence or extrapyramidal adverse events during treatment with current antipsychotics, switching to olanzapine could be a suitable therapeutic option.

The results of the present study partially support a post-hoc analysis of the previous observational study to investigate safety profiles of olanzapine conducted in Japan. Although the previous observational study with a one-year follow-up period evaluated the association between baseline characteristics of patients with schizophrenia and continuation of olanzapine therapy, it included not only patients with acute schizophrenia but also chronic schizophrenia and was not limited to olanzapine monotherapy. In that study, multivariate logistic regression analyses showed significant association of four factors (longer history of schizophrenia, lower positive symptoms, higher negative symptoms, and better health-related quality of life) with continuation of olanzapine therapy, while univariate logistic regression analyses showed significant association of many factors, including older age, male gender, inpatients, longer history of schizophrenia, higher negative symptoms at baseline with continuation of olanzapine therapy [12]. In the present study, older age was identified to be associated with continuation of olanzapine monotherapy. Although missing data made it difficult to conduct multivariate regression analysis of schizophrenia history and symptom severity, our univariate regression analysis showed a longer history of schizophrenia and higher negative symptoms at baseline to be predictors for continuation of olanzapine monotherapy. 
The present study includes important clinical findings for antipsychotic monotherapy in patients with acute schizophrenia, however there are some limitations. First of all, this is a naturalistic observational study with no randomization. To ensure the study reflected real-life clinical practice, choice of antipsychotics and the dose prescribed was at the discretion of the psychiatrist, although the study protocol stated that the oral antipsychotics should be prescribed within the approved dosage and administration in Japan. The analysis was performed individually in the olanzapine monotherapy group and the non-olanzapine antipsychotic monotherapy group, and thus comparison of the identified predictors between two groups requires careful interpretation. Secondly, patients who were lost to follow-up or changed their residence were also considered as "discontinuations" in the present study. As the follow-up period of the present study was limited to 12 months, identified predictors in the present study might not predict adherence in longer treatment. In the present study, all possible baseline characteristics that affected discontinuation may not have been collected, and missing data of the collected characteristics might have affected our results.

\section{Conclusion}

The present study provided clinical information about the predictors of adherence for antipsychotic treatment in patients with acute schizophrenia in the routine clinical practice setting. Our results indicated that the outpatient status, older age, and prior use of antipsychotics have better adherence to antipsychotic treatment. Older age, prior use of antipsychotics, and use of anti-Parkinson agents, might predict better adherence of olanzapine monotherapy.

\section{Acknowledgements}

Authors thank all the patients and clinical practitioners who participated in this study. This study was sponsored by Eli Lilly Japan K.K. (Kobe, Japan). Medical writing service was provided by Koki Yamashita, Ph.D. of Maxwell International, Inc. (Chiba, Japan) and was funded by Eli Lilly Japan K.K.

\section{Disclosure}

SF, JF and LA are employees of Eli Lilly Japan K.K. MI have received honoraria from Eli Lilly Japan K.K.

\section{References}

[1] American Psychiatric Association (2013) Diagnostic and Statistical Manual of Mental Disorders. 5th Edition, American Psychiatric Publishing, Arlington.

[2] Davis, S.M., Stroup, T.S., Koch, G.G., Davis, C.E., Rosenheck, R.A. and Lieberman, J.A. (2011) Time to All-Cause Treatment Discontinuation as the Primary Outcome in the Clinical Antipsychotic Trials of Intervention Effectiveness (CATIE) Schizophrenia Study. Statistics in Biopharmaceutical Research, 3, 253-265. http://dx.doi.org/10.1198/sbr.2011.10013

[3] Lieberman, J.A., Stroup, T.S., McEvoy, J.P., Swartz, M.S., Rosenheck, R.A., Perkins, D.O., Keefe, R.S., Davis, S.M., Davis, C.E., Lebowitz, B.D., Severe, J. and Hsiao, J.K., for the Clinical Antipsychotic Trials of Intervention Effectiveness (CATIE) Investigators (2005) Effectiveness of Antipsychotic Drugs in Patients with Chronic Schizophrenia. The New England Journal of Medicine, 353, 1209-1223. http://www.nejm.org/doi/full/10.1056/NEJMoa051688

[4] Liu-Seifert, H., Osuntokun, O.O. and Feldman, P.D. (2012) Factors Associated with Adherence to Treatment with Olanzapine and Other Atypical Antipsychotic Medications in Patients with Schizophrenia. Comprehensive Psychiatry, 53, 107-115. http://dx.doi.org/10.1016/j.comppsych.2010.12.003

[5] Tunis, S.L., Faries, D.E., Nyhuis, A.W., Kinon, B.J., Ascher-Svanum, H. and Aquila, R. (2006) Cost-Effectiveness of Olanzapine as First-Line Treatment for Schizophrenia: Results from a Randomized, Open-Label, 1-Year Trial. Value Health, 9, 77-89. http://dx.doi.org/10.1111/j.1524-4733.2006.00083.x

[6] Hodgson, R., Belgamwar, R., Al-Tawarah, Y. and MacKenzie, G. (2005) The Use of Atypical Antipsychotics in the Treatment of Schizophrenia in North Staffordshire. Human Psychopharmacology, 20, 141-147.

[7] Jung, S.H., Kim, W.H., Choi, H.J., Kang, M.H., Lee, J.S., Bae, J.N. and Kim, C.E. (2011) Factors Affecting Treatment Discontinuation and Treatment Outcome in Patients with Schizophrenia in Korea: 10-Year Follow-Up Study. Psychiatry Investigation, 8, 22-29. http://dx.doi.org/10.4306/pi.2011.8.1.22

[8] Mullins, C.D., Obeidat, N.A., Cuffel, B.J., Naradzay, J. and Loebel, A.D. (2008) Risk of Discontinuation of Atypical Antipsychotic Agents in the Treatment of Schizophrenia. Schizophrenia Research, 98, 8-15. 
http://dx.doi.org/10.1016/j.schres.2007.04.035

[9] Gaebel, W., Riesbeck, M., von Wilmsdorff, M., Burns, T., Derks, E.M., Kahn, R.S., Rössler, W. and Fleischhacker, W.W., for the EUFEST Study Group (2010) Drug Attitude as Predictor for Effectiveness in First-Episode Schizophrenia: Results of an Open Randomized Trial (EUFEST). European Neuropsychopharmacology, 20, 310-316. http://dx.doi.org/10.1016/j.euroneuro.2010.02.001

[10] Gianfrancesco, F., Rajagopalan, K., Sajatovic, M. and Wang, R.H. (2006) Treatment Adherence among Patients with Schizophrenia Treated with Atypical and Typical Antipsychotics. Psychiatry Research, 144, 177-189. http://dx.doi.org/10.1016/j.psychres.2006.02.006

[11] Kreyenbuhl, J., Slade, E.P., Medoff, D.R., Brown, C.H., Ehrenreich, B., Afful, J. and Dixon, L.B. (2011) Time to Discontinuation of First- and Second-Generation Antipsychotic Medications in the Treatment of Schizophrenia. Schizophrenia Research, 131, 127-132. http://dx.doi.org/10.1016/j.schres.2011.04.028

[12] Ye, W., Ascher-Svanum, H., Tanji, Y., Flynn, J.A. and Takahashi, M. (2011) Predictors of Continuation with Olanzapine during the 1-Year Naturalistic Treatment of Patients with Schizophrenia in Japan. Patient Prefer Adherence, 5, 611-617. http://dx.doi.org/10.2147/PPA.S26002

[13] Takahashi, M., Fujikoshi, S., Nakahara, N. and Iyo, M. (2013) The Continuation Rate of Monotherapy with Olanzapine or Other Antipsychotic Drugs in Patients with Acute-Phase Schizophrenia-A 1-Year Observational Study in Routine Clinical Practice. Japanese Journal of Clinical Psychopharmacology, 16, 1649-1660.

[14] San, L., Arranz, B., Perez, V., Safont, G., Corripio, I., Ramirez, N., Dueñas, R. and Alvarez, E. (2012) One-Year, Randomized, Open Trial Comparing Olanzapine, Quetiapine, Risperidone and Ziprasidone Effectiveness in Antipsychotic-Naive Patients with a First-Episode Psychosis. Psychiatry Research, 200, 693-701. http://dx.doi.org/10.1016/j.psychres.2012.07.005

[15] Beasley Jr., C.M., Stauffer, V.L., Liu-Seifert, H., Taylor, C.C., Dunayevich, E. and Davis, J.M. (2007) All-Cause Treatment Discontinuation in Schizophrenia during Treatment with Olanzapine Relative to Other Antipsychotics: An Integrated Analysis. Journal of Clinical Psychopharmacology, 27, 252-258. 
Scientific Research Publishing (SCIRP) is one of the largest Open Access journal publishers. It is currently publishing more than 200 open access, online, peer-reviewed journals covering a wide range of academic disciplines. SCIRP serves the worldwide academic communities and contributes to the progress and application of science with its publication.

Other selected journals from SCIRP are listed as below. Submit your manuscript to us via either submit@scirp.org or Online Submission Portal.
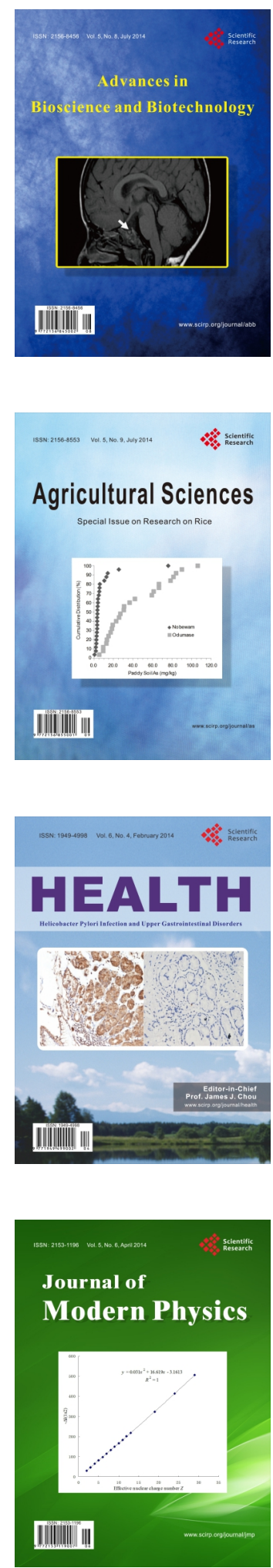
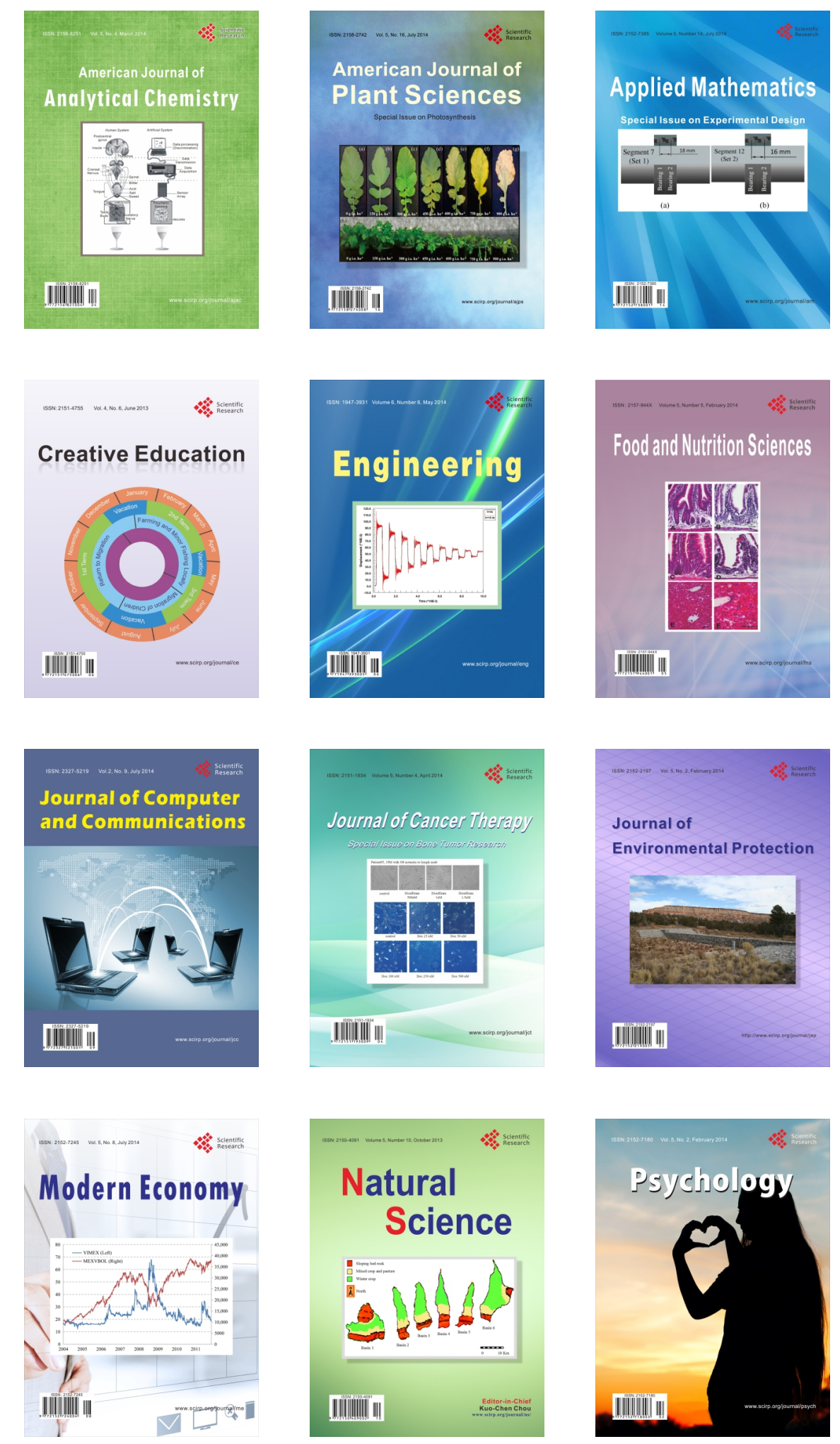